\title{
Total arterial myocardial revascularization in octogenarians
}

\author{
Anna Kędziora ${ }^{1}$, Janusz Konstanty-Kalandyk ${ }^{1,2}$, Radosław Litwinowicz' ${ }^{1}$, Piotr Mazur ${ }^{1,2}$, Bartłomiej Guzik ${ }^{2,3}$, \\ Krzysztof L. Bryniarski ${ }^{2,3}$, Jacek Piątek ${ }^{1}$ \\ ${ }^{1}$ Department of Cardiovascular Surgery and Transplantology, Institute of Cardiology, John Paul II Hospital, Krakow, Poland \\ Institute of Cardiology, Jagiellonian University Medical College, Krakow, Poland \\ ${ }^{3}$ Department of Interventional Cardiology, Institute of Cardiology, John Paul II Hospital, Krakow, Poland
}

Adv Interv Cardiol 2020; 16, 3 (61): 336-339

DOI: https://doi.org/10.5114/aic.2020.99270

\section{Introduction}

According to the current ESC/EACTS Myocardial Revascularization Guidelines, the left internal mammary artery (LIMA) should always be implanted to graft the left anterior descending (LAD) artery, but an additional arterial graft should be considered in appropriate patients undergoing coronary artery bypass grafting (CABG) [1]. In spite of inconclusive 10-year results from the Arterial Revascularization Trial (ART) [2], observational studies underline the superior patency of arterial grafts [3]. Nevertheless, based on the data published by the Society of Thoracic Surgeons (STS), bilateral internal mammary grafts are implanted in as few as $5 \%$ of CABG cases, and the use of the radial artery (RA) has declined by approximately $33 \%$ within the last decade [4].

Grafting the LIMA to the LAD is considered a gold standard in surgical revascularization. With the use of LIMA, both short and long term survival are improved in all age groups, including octogenarians $[5,6]$. However, comorbidities and limited life expectancy may potentially abolish the survival benefit from arterial revascularization in older patients. Data on arterial revascularization in octogenarians are sparse, and arterial revascularization trials omitted this age group, as the mean age of patients enrolled in the two most prominent trials (ART and RADIAL) was below 70 years old $[2,7]$.

\section{Aim}

This prompted us to assess the safety of total arterial myocardial revascularization (TAMR) in octogenarians undergoing surgical revascularization in our institution.

\section{Material and methods}

Between 2004 and 2010, 205 patients aged 80 years or older at the time of surgery underwent isolated $C A B G$ in the Department of Cardiovascular Surgery and Transplantology, John Paul II Hospital, Krakow, Poland. Of them, 7/205 (3.4\%) received TAMR, defined as implantation of arterial grafts to all narrowed coronary arteries that were intraoperatively assessed as accessible for surgical revascularization. No patients were excluded from the analysis.

Among the TAMR patients, all patients had low perioperative risk (median STS risk score: $1.4 \%$ ) and a clinical decision to use arterial grafts was made based on patients' good biological condition. Most patients were male, had three-vessel disease and left main stenosis (Table I). Coronary artery lesions were assessed as ineligible for PCI by the local Heart Team in all patients. All TAMR procedures were performed by a single experienced cardiac surgeon via median sternotomy, using cardiopulmonary bypass and blood cardioplegia. The detailed centre's CABG protocol has been previously described [5]. Additionally, at the time of data acquisition, EuroSCORE II and STS risk scores were calculated for each patient and postoperative myocardial infarction (MI) was diagnosed based on the $4^{\text {th }}$ Universal Definition of MI [8]. Perioperative complications (mortality, renal failure, stroke, prolonged ventilation, deep sternal wound infection (DSWI), reoperation, mortality and morbidity, long length of stay) were evaluated in accordance with the STS definitions [9]. In order to assess the perioperative safety of TAMR, the observed outcomes were compared with the STS risk score prediction evaluated for the study group.

In order to compare the long-term survival following TAMR and other surgical revascularization techniques, out of 205 patients aged 80 years old or older at the time of surgery, who underwent isolated CABG within the same period of time, we matched two control groups of 7 patients (LIMA to LAD combined with venous grafts

\section{Corresponding author:}

Janusz Konstanty-Kalandyk, Department of Cardiovascular Surgery and Transplantology, John Paul II Hospital, 80 Prądnicka St, $30-128$ Krakow, Poland, phone: +48 1261430 72, fax: +48 1261425 25, e-mail: jakonstanty@poczta.onet.pl

Received: 8.06.2020, accepted: 29.06.2020. 
and venous grafts only). Nearest neighbour propensity score matching with a $1: 1$ ratio was used. Patients were matched for sex, diabetes, body mass index, history of MI, CCS class on admission, left main stenosis, need for non-elective procedure, perioperative risk, and completeness of revascularization. Complete revascularization was defined as bypassing all coronary arteries in accordance with Heart Team qualification, regardless of intraoperative assessment.

Data for long-term mortality for patients in all 3 groups were obtained from the population registration office. Life expectancy was estimated for each patient in the TAMR group individually and based on the data from the Polish Central Statistical Office.

\section{Statistical analysis}

The statistical analysis was performed with IBM SPSS Statistics 25. Normal distribution was tested using the Kolmogorov-Smirnov test and continuous variables were presented as medians and interquartile ranges (IQR). The Wilcoxon signed-rank test was used to calculate the differences between predicted and observed outcomes. Kaplan-Meier survival analysis with the log-rank test was used to determine the differences between predicted and observed long-term survival.

The study was approved by the Jagiellonian University Medical College Bioethics Committee (1072.6120. 140.2019). Due to the retrospective nature of the study, the Bioethics Committee waived the necessity for signed consent.

\section{Results}

In the study group, all patients received a LIMA graft to the LAD and 5/7 (71.4\%) received complete revascularization. The right internal mammary artery (RIMA) and/or radial artery (RA) were used as additional grafts (Table I). Three $(42.9 \%)$ patients received sequential grafts. Detailed procedural data are presented in Table II. In the study group, there were no perioperative MIs or mortalities, and the observed mortality rate was lower than pre- dicted both by EuroSCORE II (0\% vs. 2.3\%) and the STS score $(0 \%$ vs. $1.4 \%)$. Prolonged ventilation was reported in 1 patient (14.3\% vs. predicted $3.9 \%$ ) and resulted in prolonged hospital stay (17 days). Detailed data on postoperative outcomes in the study group are presented in Tables III and IV.

In April 2020, after a median follow-up of 10 years, 5 (71.4\%) patients in the TAMR group remained alive. Postoperative survival was better when compared to esti-

Table I. Patient baseline characteristics and intraoperative data

\begin{tabular}{lc} 
Variable & Study population $(n=7)$ \\
\hline Baseline data: & \\
\hline Age [years] & $50(80.0-80.5)$ \\
\hline Male sex, $n(\%)$ & $26.3(23.7-29.4)$ \\
\hline BMI [kg/m²] & $55(50-60)$ \\
\hline LVEF, \% & $4(57.1)$ \\
\hline LM stenosis, $n$ (\%) & $5(71.4)$ \\
\hline Three-vessel disease, $n(\%)$ & $1(14.3)$ \\
\hline Diabetes, $n(\%)$ & $0(0)$ \\
\hline Peripheral artery disease, $n(\%)$ & $1(14.3)$ \\
\hline Chronic kidney disease, $n(\%)$ & $1(14.3)$ \\
\hline COPD, $n(\%)$ & $3(42.9)$ \\
\hline Varicose veins, $n(\%)$ & \\
\hline Intraoperative data: & $7(100)$ \\
\hline LIMA, $n(\%)$ & $2(28.6)$ \\
\hline RIMA, $n$ (\%) & $6(85.7)$ \\
\hline RA, $n(\%)$ & $3(42.9)$ \\
\hline Jump graft, $n$ (\%) & $5(71.4)$ \\
\hline Complete revascularization, $n(\%)$ & \\
\hline
\end{tabular}

$B M I$ - body mass index, LVEF - left ventricle ejection fraction, $L M$ - left main, COPD - chronic obstructive pulmonary disease, LIMA - left internal mammary artery, RIMA - right internal mammary artery, $R A$ - radial artery.

Table II. Detailed procedural data

\begin{tabular}{|c|c|c|c|c|c|c|c|}
\hline ID & Age & Sex & Mode of surgery & Procedure & CCT & CPB time & CR \\
\hline 1 & 80 & $M$ & Elective & $M g-R A+L A D-L I M A$ & 22 & 45 & $\mathrm{~N}$ \\
\hline 2 & 82 & M & Elective & Mg-RIMA + LAD-LIMA & 25 & 70 & $\mathrm{~N}$ \\
\hline 3 & 80 & $M$ & Urgent & Mg-Dg-RA (jump) + LAD-LIMA & 35 & 45 & $\mathrm{Y}$ \\
\hline 4 & 80 & $M$ & Urgent & Mg-Dg-RA (jump) + LAD-LIMA & 32 & 50 & $\mathrm{Y}$ \\
\hline 5 & 80 & M & Urgent & RCA-RA + LAD-LIMA & 22 & 50 & Y \\
\hline 6 & 80 & $\mathrm{~F}$ & Elective & LAD-Dg-LIMA (jump) + Cx-RA & 36 & 56 & Y \\
\hline 7 & 81 & $\mathrm{~F}$ & Elective & $M g-R A+R C A-R I M A+L A D-L I M A$ & 38 & 55 & $\mathrm{Y}$ \\
\hline
\end{tabular}

$M$ - male, $F$-female, CCT-cross-clamp time (minutes), CPB - cardiopulmonary bypass (minutes), CR-complete revascularization ( $Y$-yes, $N$ - no). 
Table III. Postoperative outcomes

\begin{tabular}{llcc} 
Postoperative outcome & \multicolumn{1}{c}{ Predicted $^{*}$} & Observed & $\boldsymbol{P}_{\text {-value }}{ }^{* *}$ \\
\hline Mortality & $1.407 \%(1.060-2.072)$ & $0 \%$ & 0.018 \\
\hline Renal failure & $0.862 \%(0.786-0.961)$ & $0 \%$ & 0.018 \\
\hline Stroke & $0.903 \%(0.583-1.260)$ & $0 \%$ & 0.018 \\
\hline Prolonged ventilation & $3.837 \%(3.003-5.108)$ & $14.286 \%$ & 0.018 \\
\hline Deep sternal wound infection & $0.137 \%(0.101-0.150)$ & $0 \%$ & 0.018 \\
\hline Reoperation & $1.808 \%(1.400-2.202)$ & $0 \%$ & 0.018 \\
\hline Mortality and morbidity & $6.722 \%(5.708-8.277)$ & $14.286 \%$ & 0.028 \\
\hline Long length of stay & $3.005 \%(2.498-3.229)$ & $14.286 \%$ & 0.018
\end{tabular}

*Prediction estimated based on the STS risk score; medians and interquartile ranges presented. ${ }^{* * W i l c o x o n}$ signed-rank test.

Table IV. Propensity score matching model (AUC $=0.862 \pm 0.06$; Hosmer-Lemeshow test $p=0.779$ )

\begin{tabular}{|c|c|c|c|c|}
\hline Variable & $\begin{array}{l}\text { Study group } \\
\quad(n=7)\end{array}$ & $\begin{array}{l}\text { Unmatched } \\
(n=205)\end{array}$ & $\begin{array}{l}\text { Matched LIMA (LAD) com- } \\
\text { bined with venous grafts } \\
(n=7)\end{array}$ & $\begin{array}{l}\text { Matched venous grafts } \\
\text { only } \\
(n=7)\end{array}$ \\
\hline Male sex,\% & 71.4 & 68.3 & 100 & 57.1 \\
\hline $\mathrm{BMI}\left[\mathrm{kg} / \mathrm{m}^{2}\right]^{*}$ & 26.3 & 26.5 & 25.7 & 26.7 \\
\hline Diabetes, \% & 14.3 & 28.8 & 14.3 & 28.6 \\
\hline History of MI, \% & 57.1 & 77.6 & 57.1 & 85.7 \\
\hline LM stenosis, \% & 57.1 & 51.7 & 57.1 & 57.1 \\
\hline CCS class on admission* & 3 & 3 & 2 & 3 \\
\hline Non-elective procedure, \% & 42.9 & 33.7 & 14.3 & 28.6 \\
\hline logistic EuroSCORE* & 1.1 & 1.4 & 1.2 & 1.2 \\
\hline Complete revascularization & 71.4 & 60 & 42.9 & 42.9 \\
\hline
\end{tabular}

*Median value is presented.

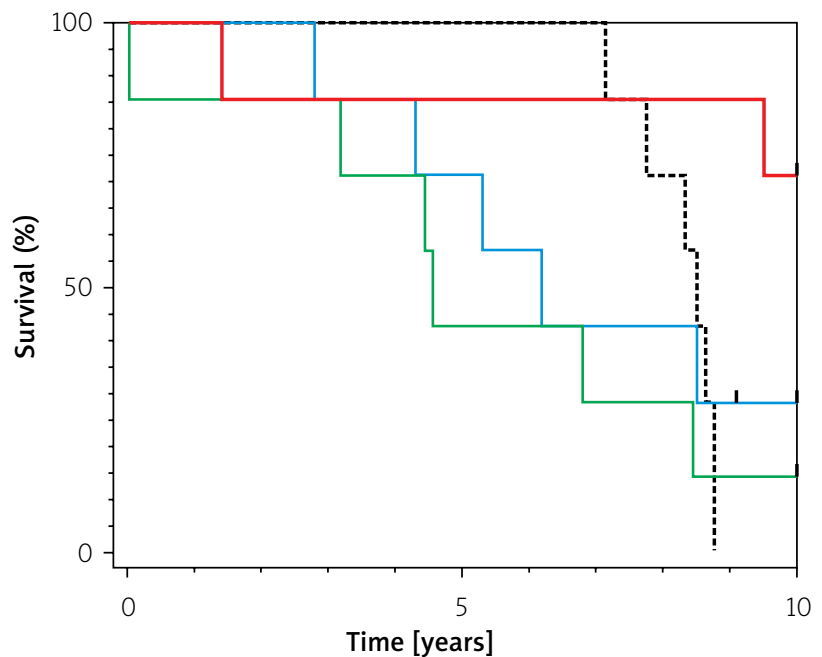

I TAMR ---L.. Life expectancy

I LIMA(LAD) + venous grafts I Venous grafts

$p=0.005$; TAMR vs. life expectancy

$p=0.026$; TAMR vs. LIMA $(\mathrm{LAD})+$ venous grafts

$p=0.090 ;$ TAMR vs. venous grafts

Figure 1. Kaplan-Meier 10-year survival mated life expectancy $(p=0.005)$ and to LIMA (LAD) combined with the venous graft group $(p=0.026)$ (Figure 1$)$.

\section{Discussion}

Despite advanced patients' age, the reported 10-year survival was comparable with the ART trial population, which comprised younger individuals (71.4\% vs. 57\%) [2]. Of note, the studied patients had low perioperative risk, were in good biological condition, and in most cases complete revascularization was achieved. Nevertheless, after matching for clinical and procedural confounders, 10-year survival remained superior for TAMR, when compared to other surgical techniques and individualized life expectancy prediction. Lower 5-year Ml incidence was previously noted for RA grafts, in comparison to venous [7], and It cannot be excluded that the cardiovascular benefit of TAMR resulted in the observed survival benefit.

Moreover, despite previous concerns regarding the increased risk for DSWI in patients receiving bilateral internal mammary revascularization, no such events were 
observed in this study. Similarly, a low infectious complication rate was previously reported by our centre for a larger population of sexagenarians undergoing arterial revascularization [10]. However, all procedures were performed by a single, well-trained cardiac surgeon and LIMA/RIMA skeletonization was usually implemented for bilateral internal mammary grafts. This observation should be considered as consistent with the current guidelines, which emphasize the importance of the surgeon's experience in coronary procedures and the benefits of skeletonization technique [1].

There were only 7 patients who received TAMR, and no data on cardiovascular events during follow-up were available for analysis. Lack of statistical significance for long-term survival analysis was likely due to study underpowering.

\section{Conclusions}

Total arterial myocardial revascularization can be performed safely among low-risk octogenarians and improved survival is observed, in comparison with life expectancy and other surgical revascularization strategies.

\section{Acknowledgments}

This work was supported by a grant from Jagiellonian University Medical College (K/ZDS/007962).

\section{Conflict of interest}

The authors declare no conflict of interest.

\section{References}

1. Sousa-Uva M, Ahlsson A, Alfonso F, et al. 2018 ESC/EACTS Guidelines on myocardial revascularization The Task Force on myocardial revascularization of the European Society of Cardiology (ESC) and European Association for Cardio-Thoracic Surgery (EACTS) Developed with the special contribution of the European Association for Percutaneous Cardiovascular Interventions (EAPCI). Eur Heart J 2019; 40: 87-165.

2. Taggart DP, Benedetto U, Gerry S, et al. Bilateral versus single internal-thoracic-artery grafts at 10 years. N Engl J Med 2019; 380: 437-46.

3. Squiers JJ, Mack MJ. Coronary artery bypass grafting-fifty years of quality initiatives since Favaloro. Ann Cardiothorac Surg 2018; 7: 516-20.

4. D'Agostino RS, Jacobs JP, Badhwar V, et al. The Society of Thoracic Surgeons Adult Cardiac Surgery Database: 2018 update on outcomes and quality. Ann Thorac Surg 2018; 105: 15-23.

5. Piatek J, Kedziora A, Konstanty-Kalandyk J, et al. Risk factors for in-hospital mortality after coronary artery bypass grafting in patients 80 years old or older: a retrospective case-series study. Peer J 2016; 4: e2667.

6. Leavitt BJ, O'Connor GT, Olmstead EM, et al. Use of the internal mammary artery graft and in-hospital mortality and other adverse outcomes associated with coronary artery bypass surgery. Circulation 2001; 103: 507-12.
7. Gaudino M, Benedetto U, Fremes S, et al. Radial-artery or saphenous-vein grafts in coronary-artery bypass surgery. N Engl J Med 2018; 378: 2069-77.

8. Thygesen K, Alpert JS, Jaffe AS, et al. Fourth universal definition of myocardial infarction (2018). Circulation 2018; 138: e618-51.

9. Shahian DM, Jacobs JP, Badhwar V, et al. The Society of Thoracic Surgeons 2018 Adult Cardiac Surgery Risk Models: part 1 - background, design considerations, and model development. Ann Thorac Surg 2018; 105: 1411-8.

10. Konstanty-Kalandyk J, Piątek J, Rudzinski P, et al. Clinical outcome of arterial myocardial revascularization using bilateral internal thoracic arteries in diabetic patients: a single centre experience. Interact Cardiovasc Thorac Surg 2012; 15: 979-83. 